\title{
Correction to: satisfied or not? exploring the interplay of individual, country and international organization characteristics for negotiation success
}

\author{
Diana Panke ${ }^{1} \cdot$ Gurur Polat $^{1}$ • Franziska Hohlstein ${ }^{1}$ \\ Published online: 18 June 2021 \\ ○) Springer Science+Business Media, LLC, part of Springer Nature 2021
}

\section{Correction to: The Review of International Organizations (2021) 16:403-429 https://doi.org/10.1007/s11558-020-09386-x}

Apart from Table 1, the tables and figures are labelled incorrectly and/or misplaced in the printed article. This erratum provides the Tables and Figures with their correct labels and indicates where in the printed article they should have been located.

Table 2 depicts the regression results. It should have been placed on page 418 or on page 419 (instead of page 414).

Figure 1 provides information on the extent of individual outcome satisfaction in 49 IOs. It is printed on page 417 (with no label at all), but should have been placed on page 408 .

The text printed directly below the Graph printed on p. 417 refers to Fig. 4 from the online appendix and should not have been included in the text of the article at all.

The original article can be found online at https://doi.org/10.1007/s11558-020-09386-x.

Diana Panke

Diana.Panke@politik.uni

1 Albert-Ludwigs-Universität Freiburg, Belfortstr. 20, 79085 Freiburg, Germany 
Table 2 Regression Analysis

\begin{tabular}{|c|c|c|c|c|c|}
\hline & Model 1 & Model 2 & Model 3 & Model 4 & Model 5 \\
\hline Workload & $\begin{array}{l}0.234 * * * \\
(0.071)\end{array}$ & $\begin{array}{l}0.192 * * \\
(0.061)\end{array}$ & & & \\
\hline Flexibility & $\begin{array}{l}0.578 * * * \\
(0.075)\end{array}$ & $\begin{array}{l}0.569 * * * \\
(0.068)\end{array}$ & $\begin{array}{l}0.613 * * * \\
(0.077)\end{array}$ & $\begin{array}{l}0.575 * * * \\
(0.075)\end{array}$ & $\begin{array}{l}0.613 \text { *** } \\
(0.077)\end{array}$ \\
\hline GDP & $\begin{array}{l}0.044 \\
(0.029)\end{array}$ & $\begin{array}{l}0.021 \\
(0.023)\end{array}$ & $\begin{array}{l}0.061 * \\
(0.028)\end{array}$ & $\begin{array}{l}0.053 \\
(0.029)\end{array}$ & $\begin{array}{l}0.061 \\
(0.028)\end{array}$ \\
\hline Government Effectiveness & $\begin{array}{l}-0.052 \\
(0.075)\end{array}$ & $\begin{array}{l}-0.001 \\
(0.068)\end{array}$ & $\begin{array}{l}0.017 \\
(0.075)\end{array}$ & $\begin{array}{l}-0.026 \\
(0.074)\end{array}$ & $\begin{array}{l}0.017 \\
(0.075)\end{array}$ \\
\hline IO Size & $\begin{array}{l}-0.004 * * \\
(0.001)\end{array}$ & $\begin{array}{l}-0.002 * \\
(0.001)\end{array}$ & & & \\
\hline Consensus Rule & & & $\begin{array}{l}0.001 \\
(0.102) \\
1.130 * \\
(0.516)\end{array}$ & & $\begin{array}{l}1.129 \\
(0.513)\end{array}$ \\
\hline Interruptions of Debates & $\begin{array}{l}-1.994 * * \\
(0.767)\end{array}$ & & & $\begin{array}{l}-1.501 * \\
(0.749)\end{array}$ & \\
\hline Extent of Arguing & $\begin{array}{l}0.478 \\
(0.317)\end{array}$ & $\begin{array}{l}0.183 \\
(0.225)\end{array}$ & & $\begin{array}{l}0.413 \\
(0.297)\end{array}$ & \\
\hline Extent of Bargaining & & & $\begin{array}{l}-0.385 \\
(0.165)\end{array}$ & & $\begin{array}{l}-0.385 \\
(0.165)\end{array}$ \\
\hline 112 & $\begin{array}{l}1.819 \\
(1.306)\end{array}$ & $\begin{array}{l}-0.657 \\
(0.976)\end{array}$ & $\begin{array}{l}-2.145 \\
(0.627)\end{array}$ & $\begin{array}{l}0.934 \\
(1.279)\end{array}$ & $\begin{array}{l}-2.144 \\
(0.652)\end{array}$ \\
\hline 213 & $\begin{array}{l}3.200 \\
(1.296) \\
5.082 * * * \\
(1.321)\end{array}$ & $\begin{array}{l}0.739 \\
(0.976) \\
2.675^{* *} \\
(0.989)\end{array}$ & $\begin{array}{l}-0.723 \\
(0.627) \\
1.196 \\
(0.641)\end{array}$ & $\begin{array}{l}2.305 \\
(1.268) \\
4.173 * * \\
(1.288)\end{array}$ & $\begin{array}{l}-0.723 \\
(0.625) \\
1.197 \\
(0.625)\end{array}$ \\
\hline 314 & $\begin{array}{l}5.082 * * * \\
7.667 * * *\end{array}$ & $\begin{array}{l}2.675 \\
5.155^{* * *} *\end{array}$ & $\begin{array}{l}1.196 \\
3.766^{* * * *}\end{array}$ & $\begin{array}{l}4.173 * * \\
6.713 * * *\end{array}$ & $\begin{array}{l}1.197 \\
3.766^{* * *}\end{array}$ \\
\hline 415 & $\begin{array}{l}7.667 * * * \\
(1.321)\end{array}$ & $\begin{array}{l}5.155^{* * *} \\
(0.989)\end{array}$ & $\begin{array}{l}3.766^{* * * *} \\
(0.641)\end{array}$ & $\begin{array}{l}6.713 \text { *** } \\
(1.288)\end{array}$ & $\begin{array}{l}3.766^{* * *} \\
(0.639)\end{array}$ \\
\hline Log Likelihood & -856.186 & -1083.959 & -851.009 & -868.340 & -851.009 \\
\hline AIC & 1736.373 & 2189.918 & 1724.018 & 1756.681 & 1722.018 \\
\hline $\mathrm{BIC}$ & 1791.139 & 2242.646 & 1774.2014 & 1802.376 & 1767.642 \\
\hline Num. obs & 709 & 892 & 708 & 713 & 708 \\
\hline Group (IOs) & 32 & 49 & 38 & 32 & 38 \\
\hline
\end{tabular}

IO random effect with $* \mathrm{p}<0.05, * * \mathrm{p}<0.01, * * * \mathrm{p}<0.001$ 


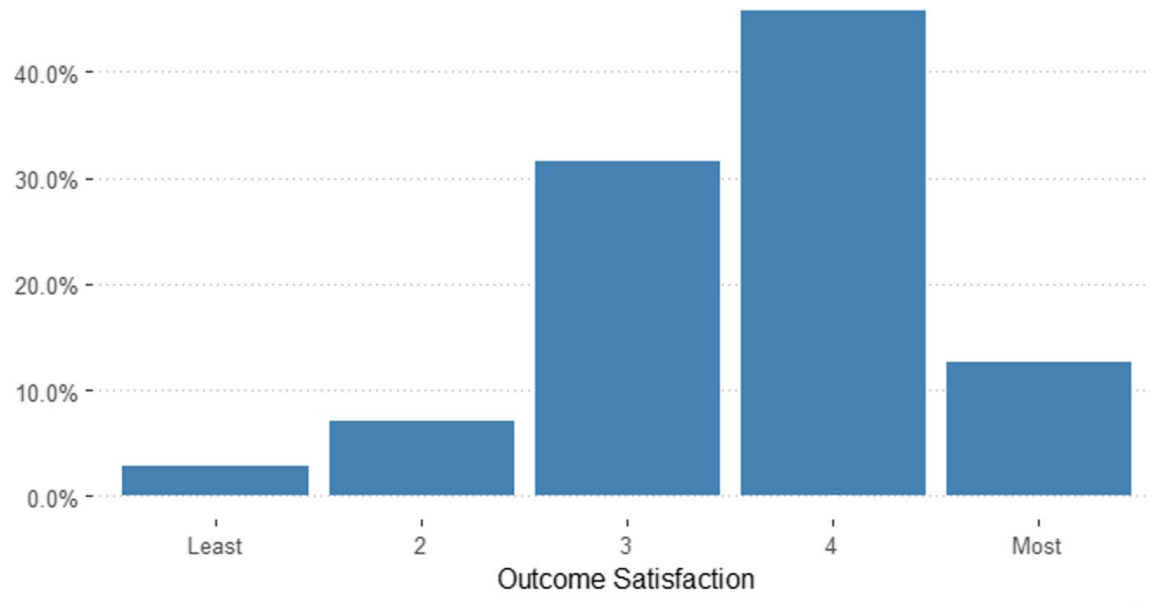

N:997

Fig. 1 Extent of individual outcome satisfaction in 49 IOs

Figure 2 shows the levels of outcome satisfaction over 49 IOs. It should have been located on page 409 (instead of being placed on page 418). Also, the labelling on page 418 is incorrect as it suggests that this is Fig. 1 instead of Fig. 2. Moreover, the figure labelled Fig. 2 on page 418 in the article is actually Fig. 3.

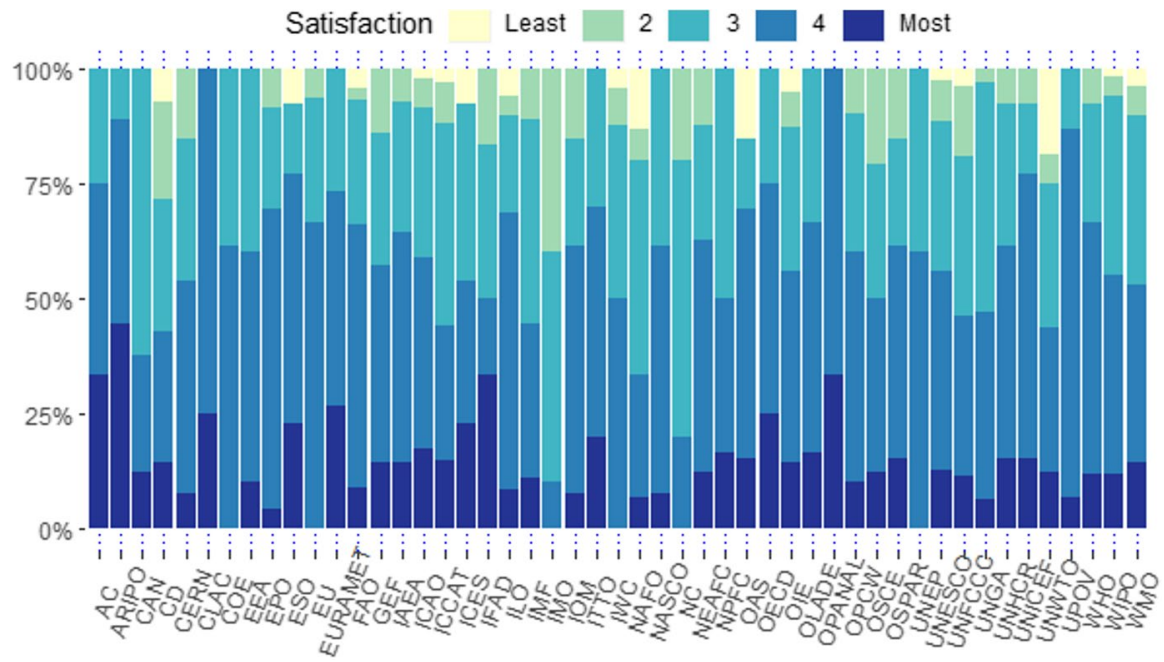

Fig. 2 Levels of outcome satisfaction over 49 IOs

Figure 3 depicts the marginal plots for high outcome satisfaction and should have been printed either on page 421 instead of page 418 . Moreover, on page 418, Fig. 3 is incorrectly labelled as Fig. 2. 


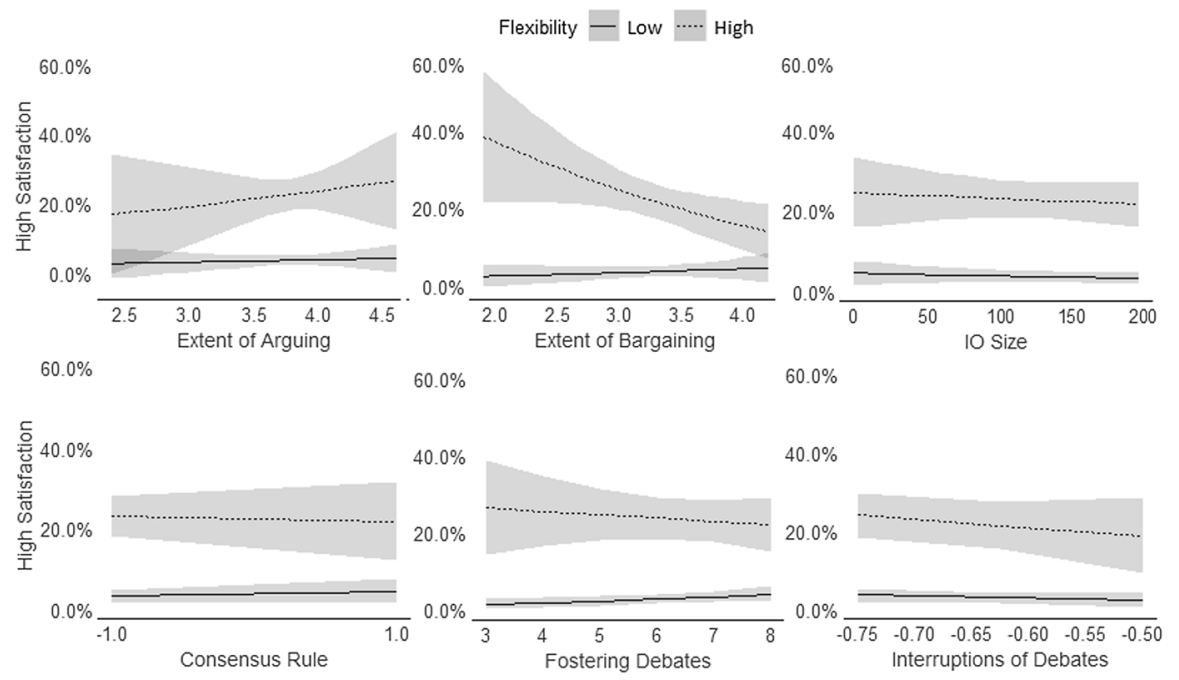

Fig. 3 The marginal plots on high outcome satisfaction (HI 1-6)

The graph printed on page 419 of the article is incorrectly labelled as Fig. 3. It displays the marginal plots on moderate outcome satisfaction, which is indeed Fig. 4 from the online appendix.

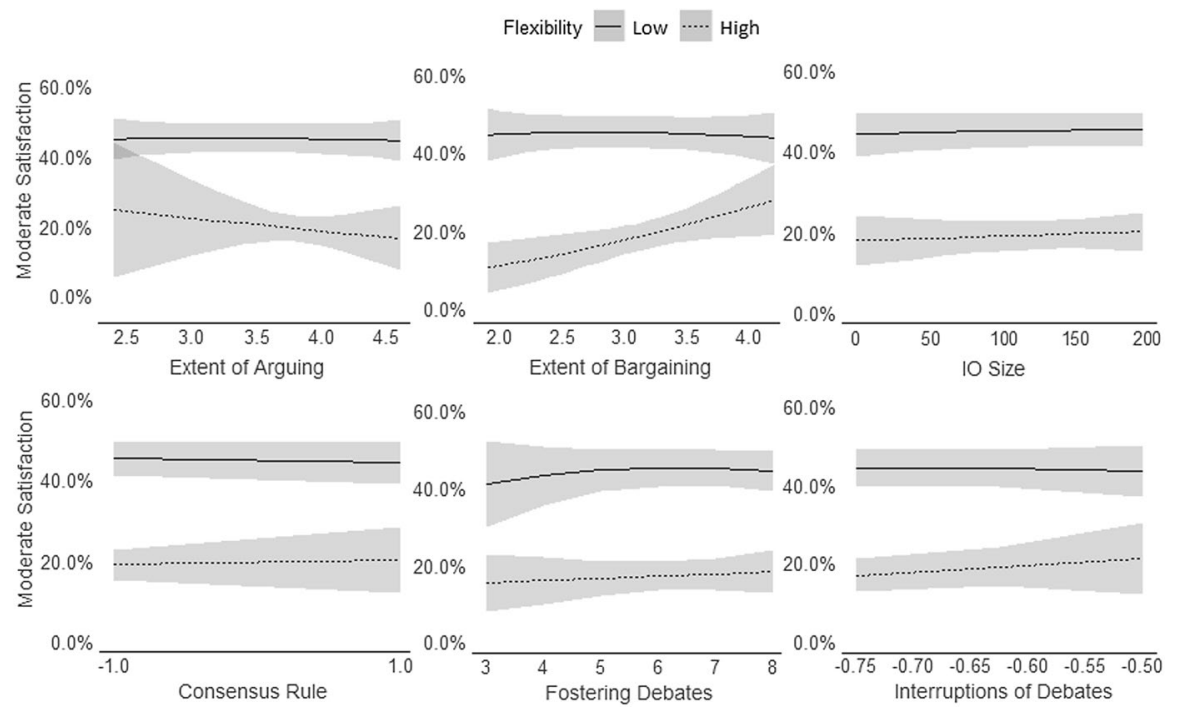

Fig. 4 The marginal plots on moderate outcome satisfaction

Publisher's Note Springer Nature remains neutral with regard to jurisdictional claims in published maps and institutional affiliations. 